Article

\title{
Optimal Allocation of Thermal-Electric Decoupling Systems Based on the National Economy by an Improved Conjugate Gradient Method
}

\author{
Shuang Rong ${ }^{1, *}$, Weixing $\mathrm{Li}^{1}{ }^{1} *$, Zhimin $\mathrm{Li}^{1}{ }^{1}$, Yong Sun ${ }^{2}$ and Taiyi Zheng ${ }^{2}$ \\ Received: 27 October 2015; Accepted: 23 December 2015; Published: 29 December 2015 \\ Academic Editor: Ling Bing Kong \\ 1 Department of Electrical Engineering, Harbin Institute of Technology, Harbin 150001, China; \\ lizhimin@hit.edu.cn \\ 2 State Grid Jilinsheng Electric Power Supply Company, Changchun 130021, China; \\ sunyong_hit@163.com (Y.S.); zhengty_j1@163.com (T.Z.) \\ * Correspondence: rongshuang@aliyun.com (S.R.); wxli@hit.edu.cn (W.L.); Tel.: +86-451-8623-0549 (S.R.); \\ +86-451-8641-3641 (W.L.)
}

\begin{abstract}
Aiming to relieve the large amount of wind power curtailment during the heating period in the North China region, a thermal-electric decoupling (TED) approach is proposed to both bring down the constraint of forced power output of combined heat and power plants and increase the electric load level during valley load times that assist the power grid in consuming more wind power. The operating principles of the thermal-electric decoupling approach is described, the mathematical model of its profits is developed, the constraint conditions of its operation are listed, also, an improved parallel conjugate gradient is utilized to bypass the saddle problem and accelerate the optimal speed. Numerical simulations are implemented and reveal an optimal allocation of TED which with a rated power of $280 \mathrm{MW}$ and $185 \mathrm{MWh}$ heat storage capacity are possible. This allocation of TED could bring approximately 16.9 billion Yuan of economic profit and consume more than $80 \%$ of the surplus wind energy which would be curtailed without the participation of TED. The results in this article verify the effectiveness of this method that could provide a referential guidance for thermal-electric decoupling system allocation in practice.
\end{abstract}

Keywords: wind power curtailment; combine heat and power; thermal-electric decoupling; improved parallel conjugate gradient

\section{Introduction}

In contemporary China, fossil-fired power generator units account for the vast majority of gross installed capacity [1-3]. The depletion of fossil fuels and the environmental degradation caused by the excessive consumption of coal are much more serious in China than in other countries with reasonable energy source structures. Toward this end, the Chinese government has promulgated the Renewable Energy Law to encourage and support the development and the application of clean and renewable energy [4]. As a relatively mature technology, with low operation and maintenance costs and large power generation per unit, wind power has occupied a considerable proportion of the existing renewable energy power sources [5]. In addition, according to the Wind Power Development Roadmap of China for 2050, the installed capacity of wind power will reach $400 \mathrm{GW}$ and $1000 \mathrm{GW}$ by 2030 and 2050, and the proportion of wind power in the gross installed capacity will increase to $15 \%$ and $26 \%$ by 2030 and 2050 [6], respectively. In the coming decades, wind power will become one of the main power sources in China. 
Due to the strong volatility and intermittency of wind power, its anti-peak regulation characteristics emerged after integration of a large amount of wind power. Moreover, the rich wind areas are relatively concentrated and distributed in the Three North region (northeast, north and northwest) of China and due to the climate features in those areas, coal-fired combined heat and power (CHP) units account for a very high proportion of the supply of both electric and heating power during the heating season, while, rapid peaking units such as gas-fired and oil-fired units are relative insufficient. Because of the combined effect of unreasonable power source structure, the influence of low water and freezing on hydro power, rich wind periods on wind power and the restriction of "Ordering Power by Heat" policy [7], wind curtailment issues frequently occur and the amount of curtailed wind energy in the total generated wind energy becomes more and more unacceptable. Thus, how to improve the wind power consumption capacity in the Three North region during the heating period has become an important research topic.

At present, one of the main measures to enlarge the wind power consumption capacity is by introducing energy storage facilities to store electric power during the wind power surplus period. References [8-11] introduce different types of energy storage technologies for wind power consumption, such as pumped storage, compressed-air energy storage, flywheel energy storage, phase change energy storage and battery energy storage. Those kinds of energy storage technology need to transform electricity into other forms of energy during the power surplus period, then transform these other forms of energy back into electric energy to feed back to the power grid. Each transformation process would waste a certain amount of energy, and those technologies, except for pumped storage, have limitations of storage scale, technology maturity and economic cost, etc. Reference [12] targets profit maximization of CHP units and provides a comparison between pure heating supply dispatching mode and pure electric supply dispatching mode. Its results show that the system's wind power consumption capacity could be improved by optimize the dispatching mode of CHP units, while the article neglects the thermal-electric coupling relationship of CHP units. Reference [13] analyzes the economic feasibility of using electric boilers with heat storage in thermal power plants to enhance the power system's wind power consumption capacity. Reference [14] researches the wind power consumption technology by controling the heating load to match the wind power output. Reference [15] discusses using the economic leverage of electricity pricing to match the wind power output and heating demand. Utilizing heat pumps to realize the combined optimal operation between CHP units and wind power plants is introduced in reference [16].

This work based on the Thermal-electric Decoupling (TED) technology [17], which could enhance the peak shaving participation ability of CHPs to assist power systems to consume more wind power during excess wind power time intervals, establishing the mathematical model of the initial investment, annual operation and maintenance costs and annual economic and policy incomes for TED. We select the maximum economic benefit as the object function to design and optimize the planning model. An improved conjugate gradient method is utilized to avoid the problem that the traditional conjugate gradient method may converge to a saddle point. A parallel computing algorithm is used to increase the optimization speed of the improved conjugate gradient method. Numerical simulation is undertaken to prove the effectiveness of this algorithm and show the significant assistance this algorithm can provide for planning departments in the configuration of TED systems.

\section{The Thermal-Electric Coupling of TED and Its Decoupling}

\subsection{Thermal-Electric Coupling of TED}

In China's north provinces, to satisfy the electric and heating demand, combined heat and electric power generation, especially extraction turbo-generator units, are widely utilized. As shown in Figure 1, the operating zone of a typical extraction turbo-generator unit is illustrated [18] where, $P_{\max }$ and $P_{\min }$ and are the maximum and minimum electric power output, respectively. In addition, the heat power output could be interval adjustable control range from 0 to $h_{\max }$ When the unit operating on the 
line $A E$, it could be treated as a straight condensing turbo-generator unit, whereas, as a back pressure turbo-generator unit when operating on the line $D C$. By adjusting the heat extraction volume from the turbine, the typical extraction turbo-generator unit could work between straight condensing mode and back pressure mode, which means the unit could be operated within the area $A B C D E$. As the policy of "Ordering Power by Heat", a forced minimum heat power output $h_{\text {force }}$ is regulated to guarantee the thermal needs during the heating season, $P_{F}$ and $P_{G}$ are the maximum and minimum electric power output by the policy, respectively. Indeed, the extraction turbo-generator unit only works within the trapezoid area $B C G F$, during heating season. Because of the narrow of electric power output adjusting interval, the peak-shaving capability of the CHP unit is limited in the heating season to participate in wind power consumption.

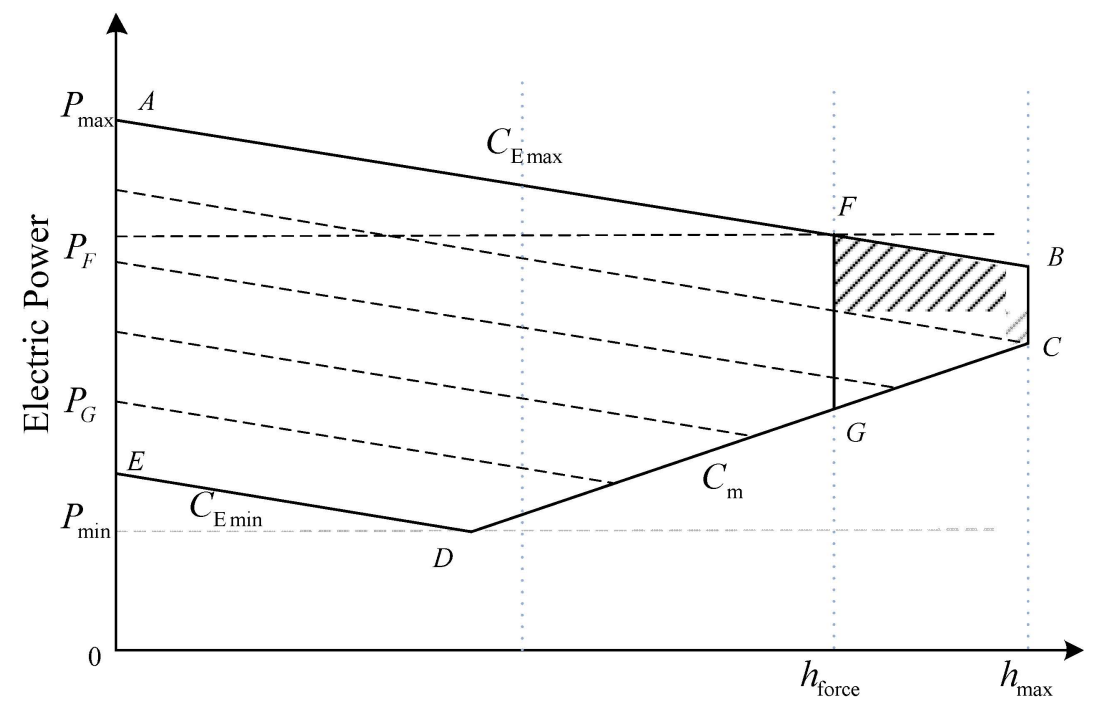

Heat Power

Figure 1. Thermal-electric coupling relationships of extraction turbo-generator units.

\subsection{Principle of Thermal-Electric Decoupling}

During the valley-load period in the heating season, because of the heating supply constraint, $\mathrm{CHP}$ units are comparatively restricted to a relatively high working condition. Due to the thermal-electric coupling of the CHP unit, the electric power output is also compelled to operate in a relatively high interval, and this shrinks the wind power acceptance spacer. Thus, there are two ways that could be adopted to enhance the capacity of wind power integration: one is to reduce CHP units' minimum required electric power outputs by introducing an outside heating resource to compensate the reduced heating power output of CHPs, and the other way is to accrete additional electric load to directly boost the electric load level during valley-load periods and superfluous wind power.

Figure 2 illustrates the schematic diagram of the thermal-electric decoupling scheme. Heat storage plays a role as an outside heat resource. The heat storage is connected with heat supply pipelines in parallel, it absorbs heat medium during peak and ordinary load periods and saves it in insulated tanks, while then feeding it back to the heat supply pipeline to compensate the reduced heating power output of CHP. The electric boiler plays the dual roles of outside heating resource and additional electric load. The electric boiler connects with the power grid and transforms the superfluous electric energy into heating energy. Due to the difference of the heat mediums of the electric boiler and heating system, a heat exchanger is necessary. The heat exchange increases the temperature of the heat medium absorbed from the heat return line by the heat generated by the electric boiler, and feeds the high temperature heat medium back to the heat supply line for the heating system. 


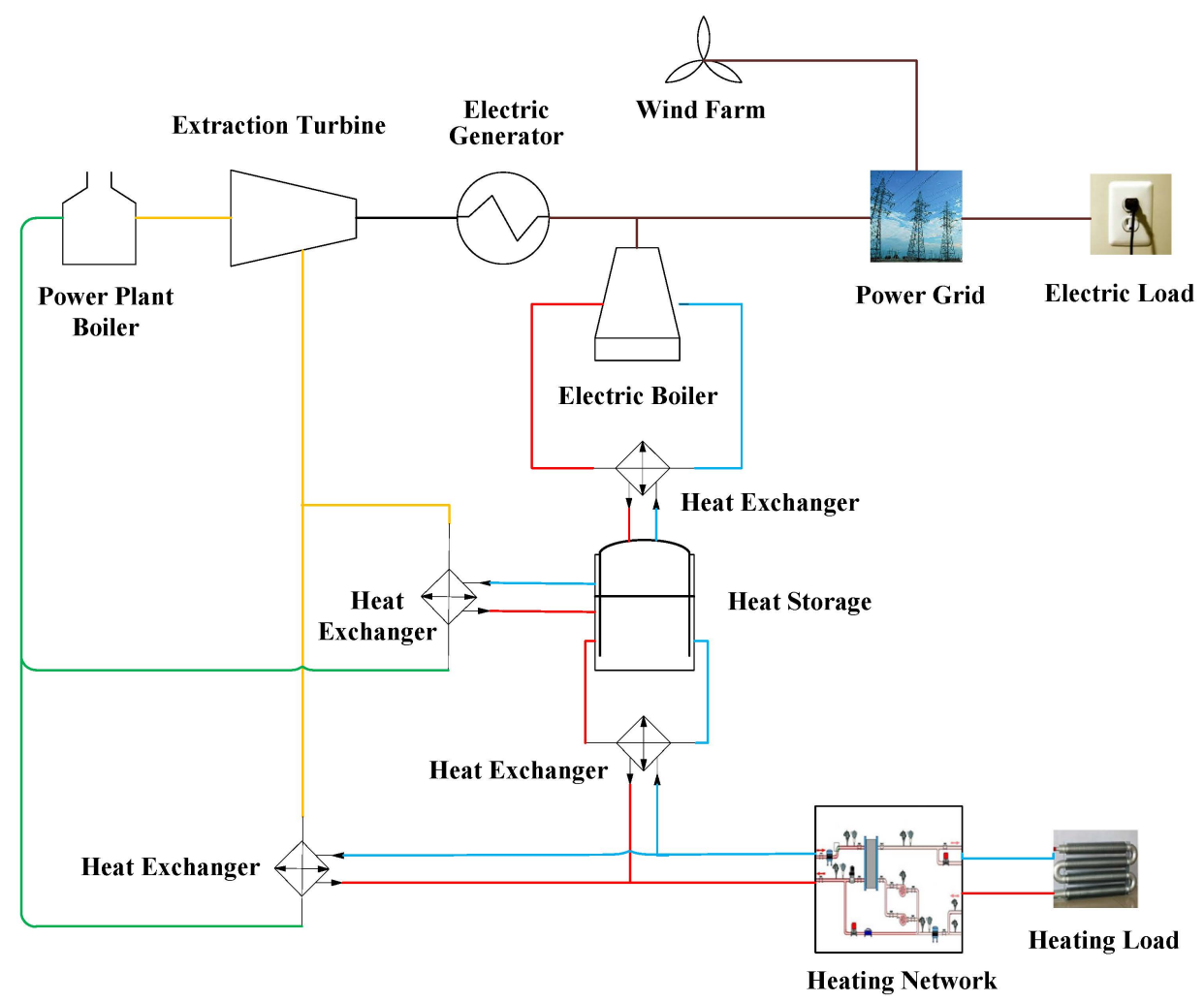

Figure 2. Schematic diagram of the thermal-electric decoupling scheme.

\section{The Economic Analysis of the Application of TED}

This paper adopts annual benefits within the design service life as the objective function:

$$
P_{\text {annual }}=B_{\text {annual }}-C_{\text {annual }}
$$

where, $P_{\text {annual }}$ is the annual benefit by the installation of TED, $B_{\text {annual }}$ is the annual incomes of TED and $C_{\text {annual }}$ is the annual cost of TED, respectively.

\subsection{The Annual Income}

The incomes of the TED application mainly comprise operating incomes such as fuel savings, and policy gains, emission reduction subsides and/or carbon trading earnings, etc. for instance:

$$
B_{\text {annual }}=B_{\mathrm{E}}+B_{H}+B_{\text {policy }}
$$

The fuel savings could be divided into savings from lower electric power output, more wind power access and lower heat power output as the participation of outside heat resources.

The equation below is the definition of annual fuel savings by lower electric power output as the participation of TED:

$$
B_{\mathrm{E}}=b_{\text {fuel }} \cdot \sum \int_{\Phi} \Delta P_{n, t} \cdot a_{n}
$$

where, respectively, $B_{E}$ is the annual fuel savings by less electricity generation, $b_{f u e l}$ is the fuel price per unit mass, $\Delta P_{n, t}$ is the reduced electric power output of CHP $n$ by TED at moment $t, a_{n}$ is the fuel consumption rate of electricity generation of $\mathrm{CHP} n, \Phi$ is path of integration for annual fuel savings. The annual fuel savings by reducing heat supply could be defined as follows:

$$
B_{\mathrm{H}}=b_{\text {fuel }} \cdot \sum \int_{\Phi} \Delta H_{n, t} \cdot b_{n}
$$


where, $B_{H}$ is the annual fuel saving by less heat supply, $\Delta H_{n, t}$ is the decreased heat power output of $\mathrm{CHP} n$ by TED, $b_{n}$ is the fuel consumption rate of heat generation of $\mathrm{CHP} n$, respectively.

The annual policy incomes is defined as:

$$
B_{\text {policy }}=b_{\text {policy }} \cdot \Gamma \cdot\left(a_{n} \cdot \sum_{n} \int_{\Phi} \Delta P_{n, t}+b_{n} \cdot \sum_{n} \int_{\Phi} \Delta H_{n, t}\right)
$$

where, $B_{\text {policy }}$ is the annual policy incomes by the utilization of TED, $b_{\text {policy }}$ is the per unit income of emission reduction under relative policy support, $\Gamma$ is the emission quantity per unit mass fuel burning.

\subsection{The Annual Cost during Design Lifetime}

The annual cost includes the annual investment cost and annual operation cost:

$$
C_{\text {annual }}=C_{\text {ttl.annual }}+C_{\text {op.annual }}=\frac{C_{\mathrm{ttl}} \cdot R_{\text {owned }}}{m}+C_{\mathrm{ttl}} \cdot\left(1-R_{\text {owned }}\right) \cdot \frac{D_{\text {bank }}\left(1+D_{\text {bank }}\right)^{m}}{\left(1+D_{\text {bank }}\right)^{m}-1}+C_{\text {op.annual }}
$$

where, $C_{\mathrm{ttl} \text {.annual }}$ is the annual investment cost discount to present equivalent annual worth, $C_{\mathrm{op} . a n n u a l}$ is annual operation cost, $\mathrm{m}$ is the design service life of TED, $D_{\text {bank }}$ is the bank discount rate and $C_{\mathrm{ttl}}$ is the initial investment of TED. $R_{\text {owned }}$ is the owned fund rate:

$$
C_{\mathrm{ttl}}=C_{\mathrm{boiler}}+C_{\mathrm{storage}}+C_{\mathrm{acc}}+C_{\mathrm{inst}}+C_{\text {trans }}+C_{\mathrm{ws}}
$$

The initial investment of TED includes the investment on electric boilers $C_{\text {boiler }}$, the cost of heat storage $C_{\text {storage, }}$ the attached facilities $C_{\text {acc }}$, system installation and commissioning cost $C_{\text {inst }}$, the investment on electric transformation equipment $C_{\text {trans }}$ and the construction cost of the workshops $C_{\mathrm{ws}}$ :

$$
C_{\text {boiler }}=b_{\text {boiler }} \cdot P_{\text {boiler }}
$$

$b_{\text {boiler }}$ is the boiler price per unit power:

$$
C_{\text {storage }}=b_{\text {cap }} \cdot V_{\text {cap }}=b_{\text {cap }} \cdot \frac{E_{\text {sto }}}{g \cdot c \cdot \rho_{\text {sto }} \cdot \Delta T}
$$

$b_{c a p}$ is the heat storage price per unit volume, $V_{c a p}$ is the volume of heat storage, $c$ is the specific heat capacity of the heat storage medium, $\rho_{\text {sto }}$ is the density of heat storage medium and $\Delta T$ is the temperature difference of the heat storage medium between output and feedback, respectively.

Assuming the cost of system attached facilities accounts for $10 \%$ of the investment on electric boilers and heat storages:

$$
C_{\text {acc }}=10 \% \cdot\left(C_{\text {boiler }}+C_{\text {storage }}\right)
$$

Assuming the cost of system installation and commissioning accounts for $30 \%$ of the investment on electric boilers, heat storages and attached facilities:

$$
C_{\text {inst }}=30 \% \cdot\left(C_{\text {boiler }}+C_{\text {storage }}+C_{\text {acc }}\right)
$$

Assuming the capacity need of system electric transformer is 1.2 times of the rated power of the boilers and pumps. Let the sum of the pumps' power equals to $3 \%$ of the $P_{b o i l e r}$ and $P_{\text {sto }}$ :

$$
C_{\text {trans }}=b_{\text {trans }} \cdot(1+20 \%) \cdot\left(P_{\text {boiler }}+\sum P_{\text {pump }}\right)=b_{\text {trans }} \cdot 1.2 \cdot\left(P_{\text {boiler }}+0.03 \cdot P_{\text {boiler }}+0.03 \cdot P_{\text {sto }}\right)
$$

Letting the occupied area per unit volume of heat storage is $1.5 \mathrm{~m}^{2}$, and assuming the area of the workshop is approximately equal to the occupied area of the heat storage:

$$
C_{\mathrm{ws}}=b_{\mathrm{ws}} \cdot A_{\mathrm{ws}}=b_{\mathrm{ws}} \cdot 1.5 \cdot V_{\mathrm{cap}}
$$

According to Equation (5) to Equation (11), the initial investment of TED $C_{\mathrm{ttl}}$ could be rewritten as below:

$$
C_{\mathrm{ttl}}=A \cdot P_{\mathrm{TED}}+B \cdot E_{\mathrm{sto}}
$$


where $\left\{\begin{array}{l}A=R_{\mathrm{TED}} \cdot\left(1.41 \cdot b_{\text {boiler }}+1.2 \cdot b_{\text {trans }}\right)+0.036 \cdot b_{\text {trans }} \\ B=\frac{1.43 \cdot b_{\text {cap }}+1.5 \cdot b_{\mathrm{ws}}}{g \cdot c \cdot \rho_{\text {sto }} \cdot \Delta T}\end{array}\right.$

The annual operation cost includes the annual productive labor cost $C_{\mathrm{HR}}$, annual system maintaining and service cost $C_{\mathrm{MM}} \& S \mathrm{~S}$ and the annual electric energy cost on system electric capacity tariff $C_{\mathrm{E}}$ :

$$
C_{\text {op.annual }}=C_{\mathrm{HR}}+C_{\mathrm{MM} \& \mathrm{SS}}+C_{\mathrm{E}}
$$

Assuming the $C_{\mathrm{HR}}$ and $C_{\mathrm{MM} \& \mathrm{SS}}$ accounts for $1 \%$ and $2 \%$ of the initial investment of TED $C_{\mathrm{ttl}}$. The annual electric capacity tariff price is defined as below:

$$
C_{\mathrm{E}}=12 \cdot b_{\text {tariff }} \cdot\left(1.2 \cdot R_{\mathrm{TED}}+0.036\right) \cdot P_{\mathrm{TED}}
$$

where, $b_{\text {tariff }}$ is the electric capacity tariff price per unit power per month.

Thus, the Equation (15) could be rewritten as:

$$
C_{\text {op.annual }}=0.3 \cdot C_{\mathrm{ttl}}+C \cdot P_{\mathrm{TED}}
$$

where, $C=12 \cdot b_{\text {tariff }} \cdot\left(1.2 \cdot R_{\mathrm{TED}}+0.036\right)$

According to Equations (4), (12) and (15). The annual cost of TED $C_{\text {annual }}$ could be abbreviated as:

$$
C_{\text {annual }}=E \cdot P_{\mathrm{TED}}+F \cdot E_{\text {sto }}
$$

where, $\left\{\begin{array}{l}E=\left(\frac{R_{\text {owned }}}{m}+\left(1-R_{\text {owned }}\right) \cdot \frac{D_{\text {bank }}\left(1+D_{\text {bank }}\right)^{m}}{(1+D)^{m}-1}+C+0.3\right) \cdot R_{\mathrm{TED}} \cdot\left(1.41 \cdot b_{\text {boiler }}+1.2 \cdot b_{\text {trans }}\right)+0.036 \cdot b_{\text {trans }} \\ F=\left(\frac{R_{\text {owned }}}{m}+\left(1-R_{\text {owned }}\right) \cdot \frac{D_{\text {bank }}\left(1+D_{\text {bank }}\right)^{m}}{\left(1+D_{\text {bank }}\right)^{m}-1}+C\right) \cdot \frac{1.43 \cdot b_{\text {cap }}+1.5 \cdot b_{\text {ws }}}{g \cdot c_{\text {sto }} \cdot \rho_{\text {sto }} \cdot \Delta T}\end{array}\right.$

\section{Constraint Conditions}

\subsection{Energy Balance Constraint}

The energy balance constraint could be classified as electric power balance and heating power balance.

Electric power balance:

$$
\sum P_{m, t}^{\mathrm{non}-\mathrm{CHP}}+\sum P_{n, t}^{\mathrm{CHP}}+\sum P_{j, t}^{\mathrm{WP}}=L_{\mathrm{E}, t}+\sum P_{k, t}^{\mathrm{TED}}
$$

where, $P_{m, t}^{\text {non-CHP }}$ is the electric power output of non-CHP unit $m$ at moment $t, P_{n, t}^{\mathrm{CHP}}$ is the electric power output of $\mathrm{CHP}$ unit $n, P_{j, t}^{\mathrm{WP}}$ is the electric power output of wind turbine $\mathrm{j}$ at moment $\mathrm{t}, L_{\mathrm{E}, t}$ is the system electric load and the power transfer to outside at the cross-section, $P_{k, t}^{\mathrm{TED}}$ is the input electric power of TED unit $k$ at moment $t$, respectively.

Heating power balance:

$$
\sum H_{z, t}^{\mathrm{HB}}+\sum H_{n, t}^{\mathrm{CHP}}+\sum H_{k, t}^{\mathrm{TED}, \text { sup }}=L_{\mathrm{Heat}, t}+\sum H_{k, t}^{\mathrm{TED}, \text { sto }}
$$

where similarly, $H_{z, t}^{\mathrm{HB}}$ is the heating power output of heating boiler unit $z$ at moment $t, H_{n, t}^{\mathrm{CHP}}$ is the heating power output of CHP unit $n, H_{k, t}^{\mathrm{TED} \text {,sup }}$ is the heating power output of TED unit $k$ at moment $t, L_{\text {Heat }, t}$ is the system heating load, $H_{k, t}^{\mathrm{TED}, \text { sto }}$ is the input heating power of TED unit $k$ at moment $t$, respectively.

\subsection{Electric Power Output and Heating Power Output Constraints}

Active power output constraint:

$$
\underline{P_{n}} \leqslant P_{n, t} \leqslant \overline{P_{n}}
$$


$\overline{P_{n}}$ and $\underline{P_{n}}$ are the upper and lower bounds of the active power output of electric power generation unit $n$.

Heating power output constraint:

$$
\underline{H_{n}} \leqslant H_{n, t} \leqslant \overline{H_{n}}
$$

$\overline{H_{n}}$ and $H_{n}$ are the upper and lower bounds of the heating power output of heat source $n$.

\subsection{The Constraint of Thermal-Electric Output Coupling of CHPs}

$$
H_{n, t} \leqslant C_{m, n} \cdot P_{n, t}+K_{n}
$$

where $C_{m, n}$ is the heating to electric power output ratio of CHP unit $n$ under backpressure condition, $K_{n}$ is a constant.

\subsection{The Constraint of Unit Ramp Rate}

$$
\left\{\begin{array}{l}
-\underline{P_{\mathrm{d}, n}} \leqslant P_{n, t}-P_{n, t-1} \leqslant \overline{P_{\mathrm{u}, n}} \\
-\underline{H_{\mathrm{d}, n}} \leqslant H_{n, t}-H_{n, t-1} \leqslant \overline{H_{\mathrm{u}, n}}
\end{array}\right.
$$

where, $\overline{P_{\mathrm{u}, n}}, P_{\mathrm{d}, n}, \overline{H_{\mathrm{u}, n}}$ and $H_{\mathrm{d}, n}$ are the upper and lower bonds of the electric power and heating power ramp rate of $\overline{\mathrm{CHP}}$ unit $n$, respectively.

\subsection{The Constraint of TED Operation}

$$
\begin{cases}\sum\left(H_{k, t}^{\mathrm{TED}, \text { sup }}-H_{k, t-1}^{\mathrm{TED}, \text { sup }}\right) \leqslant \sum \underline{H_{\mathrm{d}, n}} & \left(H_{k, t}^{\mathrm{TED}, \text { sup }} \geqslant H_{k, t-1}^{\mathrm{TED}, \text { sup }}\right) \\ \sum\left(H_{k, t-1}^{\mathrm{TED}, \text { sup }}-H_{k, t}^{\mathrm{TED}, \text { sup }}\right) \leqslant \sum \underline{H_{\mathrm{u}, n}} & \left(H_{k, t}^{\mathrm{TED}, \text { sup }} \geqslant H_{k, t-1}^{\mathrm{TED}, \text { sup }}\right)\end{cases}
$$

where, $H_{k, t}^{\mathrm{TED} \text {,sup }}$ is the heating power output of TED unit $k$ at moment $t$.

$$
\begin{cases}\left(P_{t}^{\mathrm{WP}, \mathrm{acc}}-P_{t-1}^{\mathrm{WP}, \mathrm{acc}}\right)-\sum\left(P_{k, t}^{\mathrm{TED}}-P_{k, t-1}^{\mathrm{TED}}\right) \leqslant \sum \underline{P_{\mathrm{d}, n}} \quad\left(P_{k, t}^{\mathrm{TED}} \geqslant P_{k, t-1}^{\mathrm{TED}} \cap P_{t}^{\mathrm{WP}, \mathrm{acc}} \geqslant P_{t-1}^{\mathrm{WP}, \mathrm{acc}}\right) \\ \sum\left(P_{k, t-1}^{\mathrm{TED}}-P_{k, t}^{\mathrm{TED}}\right)+\left(P_{t-1}^{\mathrm{WP}, \mathrm{acc}}-P_{t}^{\mathrm{WP}, \mathrm{acc}}\right) \leqslant \sum \underline{P_{\mathrm{u}, n}} \quad\left(P_{k, t}^{\mathrm{TED}}<P_{k, t-1}^{\mathrm{TED}} \cap P_{t}^{\mathrm{WP}, \mathrm{acc}}<P_{t-1}^{\mathrm{WP}, \mathrm{acc}}\right)\end{cases}
$$

where, $P_{t}^{\mathrm{WP}, \text { acc }}$ is the consumed wind power by grid at $k$ at moment $t . P_{k, t}^{\mathrm{TED}}$ is the electric power input of TED unit $k$ at moment $t$.

\section{Improved Parallel Conjugate Gradient Method}

With the purpose of solving the problem that the classic conjugate gradient method may converge to a saddle point during the process of unconstrained optimization [17], this work adopts an improved parallel conjugate gradient method to bypass the problem.

\subsection{Unconstrained Optimization Algorithm}

To solve unconstrained optimization problems, conjugate gradient methods are utilized frequently. For that method, if the gradient of the objective function is less than the given accuracy, the method would stop. Well, the stop of the optimization may result in two situations as the gradient at the saddle point also could be zero. One is that the optimization does converge to the optimum value, and other is the optimization converges to the saddle point. In the second situation, due to the inexistence of a negative gradient direction the optimization algorithm is forced to stop.

The improved parallel conjugate gradient method based on the conjugate gradient method, improves the stop criterion of the optimization, also, it takes the advantage of the multi-core/many-core of computer and utilizes parallel computing algorithm to boost the optimal velocity. When the 
optimization iterates to a saddle point, the IPCG adopts the directions, corresponding to all of the eigenvectors which relative to all of the positive eigenvalue of the Jacobian matrix, as the new searching directions, and uses a parallel computing algorithm to search for the optimum value in parallel.

\subsection{Parallel Computing Approach}

This work adopts a parallel computing approach to accelerate the optimization speed of the improved conjugate gradient method. The Graphics Processing Units (GPU) parallel computing approach in reference $[19,20]$ is utilized. By virtue of the very high peak computational performance offered by the massive throughput-oriented multi-core processor of GPUs, the optimization speed could be boosted remarkably.

\subsection{Algorithm Calculating Procedures}

The calculation procedure is as follows: for an unconstrained optimization problem:

$$
\min f(x), x \in R
$$

Step 1. Presenting an arbitrary initial value $x_{0} \in R$, set the initial direction:

$$
d_{0}=-\nabla f\left(x_{0}\right)
$$

Setting the required precision $\varepsilon$ and letting $k=1$.

Step 2. If $\left\|\nabla f\left(x_{k}\right)\right\| \leqslant \varepsilon$, calculate the eigenvalues of the Jacobian matrix. On condition that all of the eigenvalues are positive, the optimization converges to the optimum value and the optimal process could be stopped, while, in case of the existence of negative eigenvalues, it means the optimization converges to a saddle point $x^{\#}$; in this condition the algorithm process shifts to Step 3. Else if $\left\|\nabla f\left(x_{k}\right)\right\|>\varepsilon$, shift to Step 6.

Step 3. Calculate all of the eigenvalues of the Jacobian matrix $\lambda_{1}, \lambda_{2} \ldots \lambda_{m}$ and the corresponding eigenvectors $\gamma_{1}, \gamma_{2} \ldots \gamma_{m}$, setting a new searching point:

$$
x_{0, i}=x^{\#}+\gamma_{i}(i \in m)
$$

Set a new searching direction:

$$
d_{0, i}=-\nabla f\left(x^{\#}\right)
$$

Using parallel computing method, search in parallel for the optimum value from the new search points and new search directions. Shift to step 4.

Step 4. For $i \in m$, if $\left\|\nabla f\left(x_{0, i}\right)\right\| \leqslant \varepsilon$, it means the optimization is convergent, then judge the existence of a saddle point with the method mentioned in Step 2. If there is no saddle point, the optimization converges to the optimum value. If a saddle point could be detected, shift to Step 3 to re-set the new search point and a new direction. In case that $\left\|\nabla f\left(x_{0, i}\right)\right\| \leqslant \varepsilon$, shift to Step 5.

Step 5. Set a new search direction:

$$
d_{n, i}=-\nabla f\left(x_{n, i}\right)+\alpha_{n-1, i} \cdot d_{n-1, i}
$$

$$
\text { where } \alpha_{n-1, i}=\left\{\begin{array}{l}
0 \quad n=1 \\
\frac{\left\|\nabla f\left(x_{n, i}\right)\right\|^{2}}{\left\|\nabla f\left(x_{n-1, i}\right)\right\|^{2}} \quad n>1
\end{array}\right.
$$

Calculate the optimal step size by solving the following one-dimensional problem:

$$
\min \phi\left(\alpha_{n, i}\right)=f\left(x_{n, i}+\alpha \cdot d_{n, i}\right)
$$

Letting: 


$$
x_{n+1, i}=x_{n, i}+\alpha_{n, i} \cdot d_{n, i}
$$

Then, shift to Step 4

Step 6. Set a new searching direction:

$$
d_{k}=-\nabla f\left(x_{k}\right)+\beta_{k-1} \cdot d_{k-1}
$$

where, $\alpha_{n-1, i}= \begin{cases}0 & n=1 \\ \frac{\left\|\nabla f\left(x_{n, i}\right)\right\|^{2}}{\left\|\nabla f\left(x_{n-1, i}\right)\right\|^{2}} & n>1\end{cases}$

Calculating the optimal step size by solving the following one-dimensional problem:

$$
\min \phi\left(\alpha_{n, i}\right)=f\left(x_{n, i}+\alpha \cdot d_{n, i}\right)
$$

Letting:

$$
x_{k+1}=x_{k}+\alpha_{k} \cdot d_{k}
$$

Then, shift to Step 2.

\section{System Modeling and Numerical Simulation}

\subsection{General Situation of Simulation}

This work adopts the electric-heating hybrid system introduced in reference [18], and the economic parameters needed for economic analysis are set as in Table 1.

Table 1. The economic parameters for simulation.

\begin{tabular}{ccc}
\hline Item & Value & Unit \\
\hline Electric Boiler & 75 & $10^{4}$ Yuan per MW \\
Heat Storage & 0.7 & $10^{4}$ Yuan per $\mathrm{m}^{3}$ \\
Pumps & 0.4 & $10^{4}$ Yuan per MW \\
Electric Power Transformer Equipment & 25 & $10^{4}$ Yuan per MVA \\
Utility Building & 0.4 & $10^{4}$ Yuan per $\mathrm{m}^{2}$ \\
Fuel & 0.065 & $10^{4}$ Yuan per ton \\
Bank discount rate & 6.55 & $\%$ \\
Design service life & 20 & Year \\
Carbon trading price & 50 & Yuan per ton \\
Coal Evaluation on Electricity Generation & 0.1229 & ton per MWh \\
Coal Evaluation on Heat Generation & 0.03412 & tom per GJ \\
Heat Price & 21.5 & Yuan per GJ \\
Electricity Price & 386.4 & Yuan per MWh \\
\hline
\end{tabular}

\subsection{System Modeling}

Electric load model:

Utilizing annual system load forecasting results, annual weekly electric load curve, hourly electric load curves to simulate the prospective system load curve on time series:

$$
P_{\text {load }, t}=\left\{\begin{array}{lr}
P_{\text {load }, t}^{L}=\delta P_{\text {Eyear }} \zeta_{\mathrm{E}, n} \phi_{t} & \text { heavy load day } \\
P_{\text {load }, t}^{M}=(1-\delta) P_{\text {Eyear }} \zeta_{\mathrm{E}, n} \phi_{t} & \text { normal load day } \\
P_{\text {load }, t}^{S}=(1-2 \delta) P_{\text {Eyear }} \zeta_{\mathrm{E}, n} \phi_{t} & \text { light load day }
\end{array}\right.
$$

where, $\xi_{\mathrm{E}, n}(\mathrm{n} \in 1: 20)$ is the weekly system electric load curve (p.u.) during heating season, $\phi_{t}(\mathrm{t} \in 1: 24)$ is the hourly system load curve (p.u.), $P_{\text {Eyear }}$ is the maximum system electric load during the heating season. Assuming that there are two heavy load days, three normal load days and two light load days 
each week and the $\delta$ represents the variation factor of system load. The annual weekly electric load curve is presented in Figure 3a, the heat season starts from the 40th week and lasts to the 12th week next year.

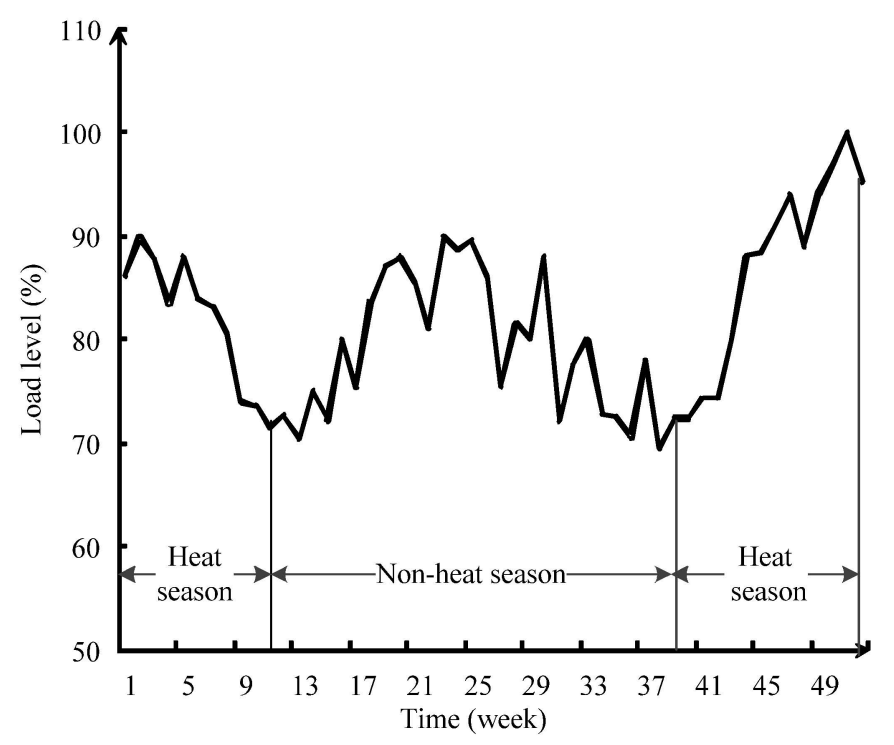

(a)

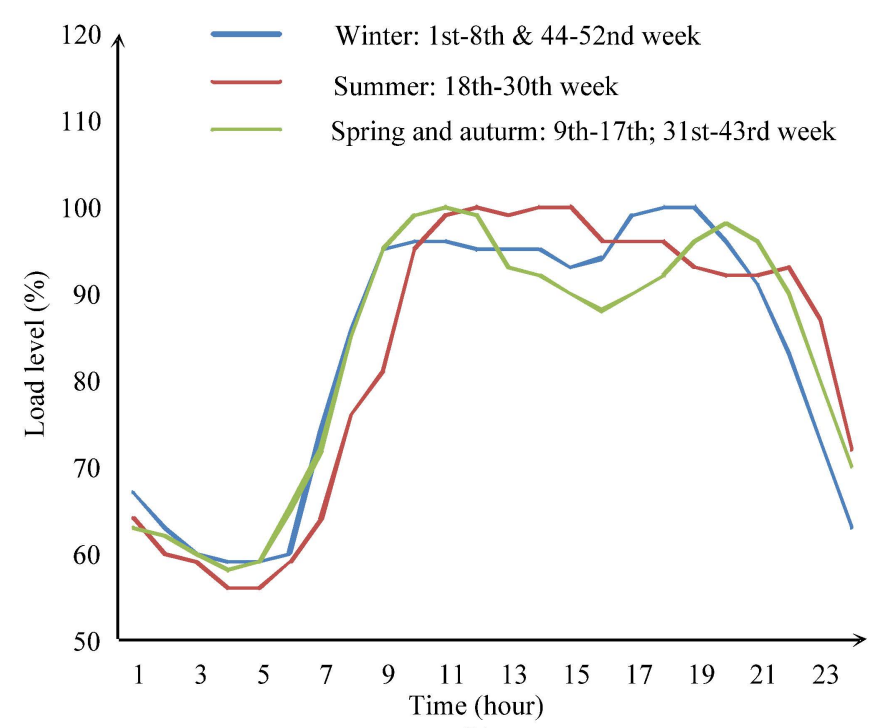

(b)

Figure 3. The weekly (a) and hourly (b) electric load curves.

From the figure, it could be discovered that the load level increases with beginning of heat season and touch the peak at the end of a year, then going down with the coming of non-heat season. Figure $3 \mathrm{~b}$ presents the hourly electric load curves for different seasons and reveals that although the time of occurrence and duration of the peak load are different from season to season, the vale load emerges and fades away at the similar period.

Heat load model:

Utilizing seasonal heat load forecasting results and weekly load curve and typical daily system to simulate the prospective heat load curve on time series:

$$
H_{n}=H_{\text {season }} \xi_{\mathrm{H}, n}
$$


where, $\xi_{\mathrm{H}, n}$ ( $\mathrm{n} \in$ heat season) is the daily heat load curve (p.u.) during heating season, $H_{\text {season }}$ is the maximum heat load during the heating season.

The daily heat load level curve is illustrated below in Figure 4, from which it could be detected that the heat load is relatively low at the beginning and ending periods of the heating season compared with deep winter as the reason of weather change.

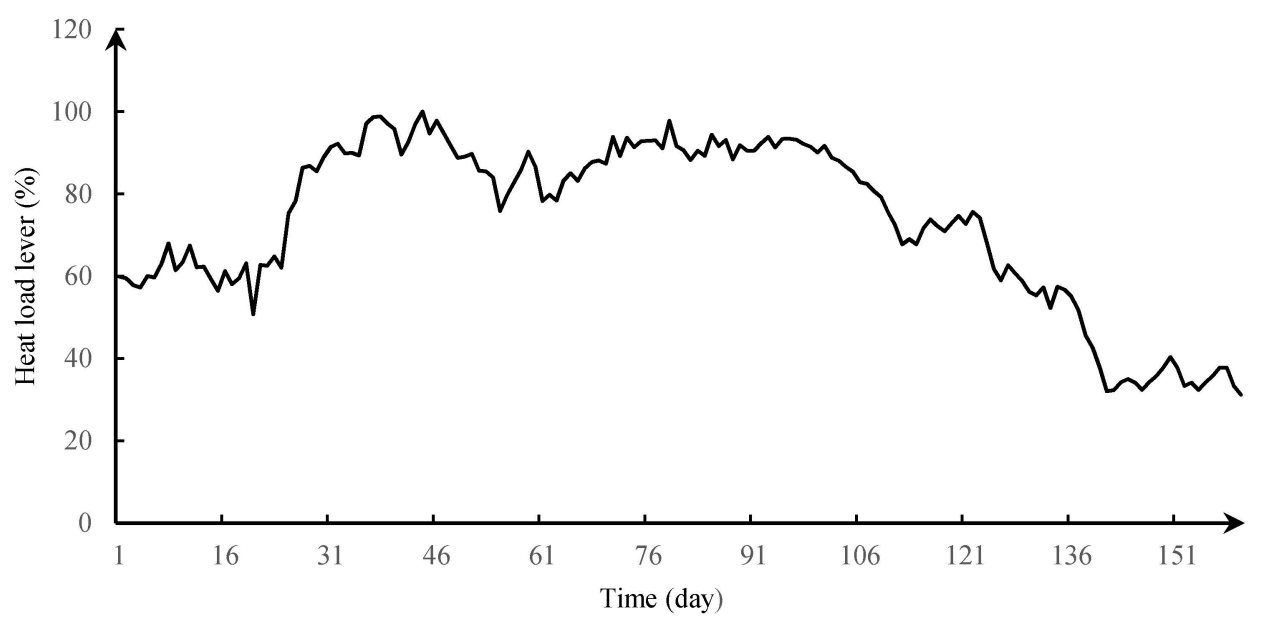

Figure 4. The daily heat load level during heating season.

In light of the heating load data we have obtained from the Sixth Subsidiary of Fuxin District Heating Group, Fuxin City (Liaoning Province, China) the hourly heating load during a typical day shows that both ends are high, and the middle is low, while, the peak-valley load difference is relatively minor, about 1.5\%, as shown in Figure 5, compared with the maximum heating load value as shown in the table and figure below.

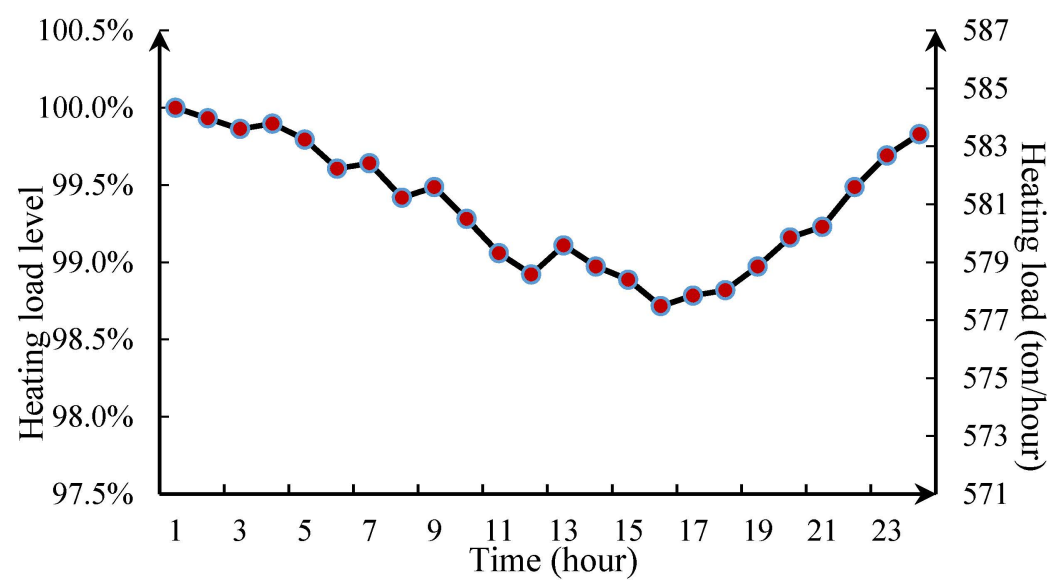

Figure 5. The hourly heating load variation of a typical heating day.

We consider that although the hourly heating needs among diverse types of heating load such as industrial heating load, municipal heating load, commercial heating load and residential heating load, are different, the complementarity among them is comparatively strong. In order to simplify the models, shorten the simulation time and without loss of generality, we use a stable and continuous model to present the hourly heating loads during a day time.

Wind farms model:

Weibull's distribution is employed to generate the hourly wind speed data, and its probability density function is as below: 


$$
f(v)=\frac{k}{c}\left(\frac{v}{c}\right)^{k-1} \exp \left[-\left(\frac{v}{c}\right)^{k}\right]
$$

where, $k$ and $c$ are the shape parameter and scale parameter of the two-parameter Weibull's distribution, respectively. The available wind power output of a single wind turbine could be calculated by non-linear equations between wind speed and wind power output below:

$$
P_{\text {wind }, t, \tau}= \begin{cases}0 & 0 \leqslant v \leqslant v_{c i} \\ \left(A+B v+C v^{2}\right) P_{r, \tau} & v_{c i} \leqslant v \leqslant v_{r} \\ P_{r, \tau} & v_{r} \leqslant v \leqslant v_{c o} \\ 0 & v_{c o} \leqslant v\end{cases}
$$

where, $v$ is the wind speed, $v_{c i}$ is cut-in wind speed, $v_{r}$ is rated wind speed, $v_{c o}$ is cut-out wind speed, $P_{\text {wind, }, \tau} \tau$ is wind power output and $P_{r}$ is rated wind power.

The power output of the wind farm could be determined as below:

$$
P_{\mathrm{WF}, t}=\alpha \sum_{\tau=1}^{N_{w}} \beta P_{\mathrm{wind}, t, \tau}
$$

where, $\alpha$ is the wake effect coefficient of wind farm. $N_{\mathrm{w}}$ is the number of wind turbines within the wind farm. If $\beta=1$, the wind turbine is working and $\beta=0$ otherwise. The Monte Carlo simulation technique is used to sample the system operating conditions under different TED configurations. The sampling objects include the wind speed probability distribution, the hourly heat curves, and the system load curves, etc. We statistically compute the consumed wind energy $E_{\text {saved }}$ by TED during the whole heating period, then apply a polynomial to fit the distribution of $E_{\text {saved }}$ under different TED configurations. The maximum likelihood estimator is as below:

$$
L\left(P_{\mathrm{TED}}, E_{\mathrm{sto}} \mid E_{\mathrm{saved}}\right)=a_{10} \cdot P_{\mathrm{TED}}+a_{20} P_{\mathrm{TED}}{ }^{2}+a_{30} P_{\mathrm{TED}}{ }^{3}+a_{01} \cdot E_{\mathrm{sto}}+a_{02} \cdot E_{\mathrm{sto}}{ }^{2}+a_{03} \cdot E_{\mathrm{sto}}{ }^{3}+a_{11} \cdot P_{\mathrm{TED}} \cdot E_{\mathrm{sto}}+a_{12} \cdot P_{\mathrm{TED}} \cdot E_{\mathrm{sto}}{ }^{2}+a_{21} \cdot P_{\mathrm{TED}}{ }^{2} \cdot E_{\mathrm{sto}}+C
$$

\subsection{Simulation Results}

Different scenarios are setting to discuss the impacts of TED configuration on the annual profits of TED. Table 2 presents the configuration cases.

Table 2. The configuration of power outputs under different scenarios.

\begin{tabular}{cc}
\hline Scenario & The Ratio of between $\boldsymbol{P}_{\text {sto }}$ and $\boldsymbol{P}_{\text {boile }}$ \\
\hline A & $5: 35$ \\
B & $10: 30$ \\
C & $15: 25$ \\
D & $20: 20$ \\
E & $25: 15$ \\
F & $30: 10$ \\
G & $35: 5$ \\
\hline
\end{tabular}

Figure 6 presents the contour map of annual profits under scenario E, from which it could be discovered that the global optimum value is located at point $\mathrm{A}$, while a local optimum value exists at point $B$ which may lead traditional conjugate gradient methods to converge to this value which is not the global optimal. Three points, $\alpha(80,120) \beta(80,420)$ and $\gamma(480,420)$, are selected as optimal initial points to verify the effectiveness of improved parallel conjugate gradient method. The results show that with the conditional conjugate gradient method, the optimization could converge to the global optimum value A from initial point $\beta$ and $\gamma$, but converges to the local optimum value B from point $\alpha$, 
while, with improved parallel conjugate gradient method, the optimization could converge to A from all initial points.

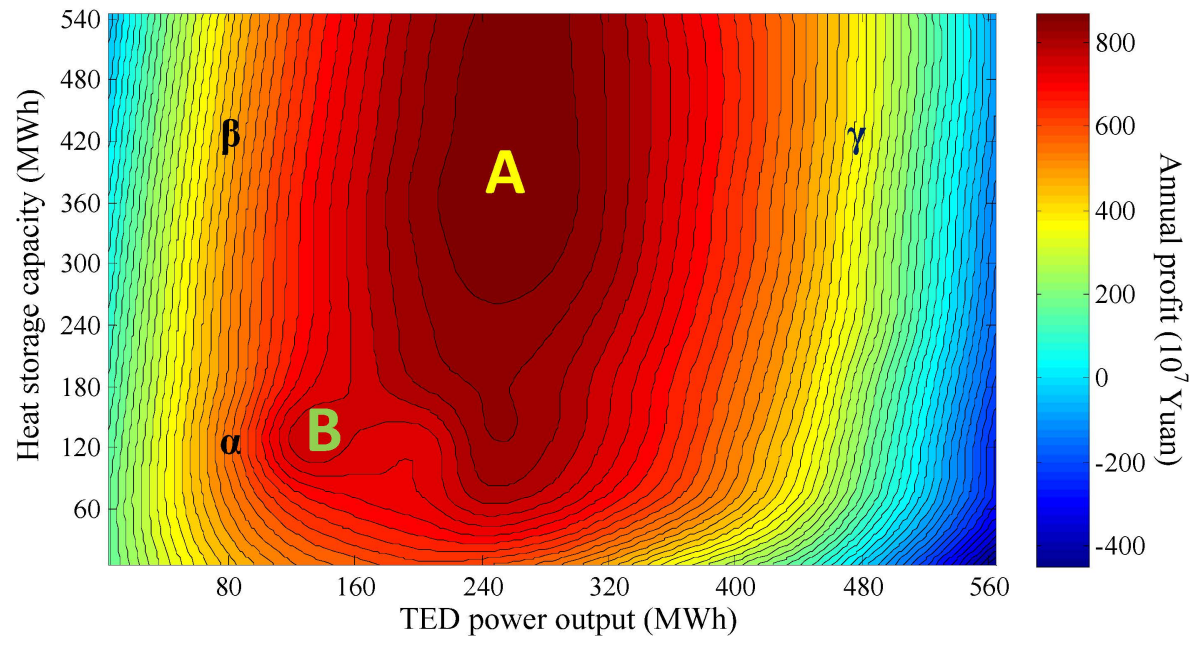

Figure 6. The contour map of annual profits under scenario E.

Table 3 express the parallel computing speedup ratio under different scenarios. From the table, it could be discover that the acceleration effect of parallel computing is not obvious under scenarios $B$ and $C$, it reason being that the saddle point does not appear in those scenarios, while, for other scenarios where a local optimum value and total computation time exist, the speedup ratio is considerable.

Table 3. The speedup ratio of parallel computing.

\begin{tabular}{cccc}
\hline \multirow{2}{*}{ Scenario } & \multicolumn{2}{c}{ Computation Time (ms) } & \multirow{2}{*}{ Speedup Ratio } \\
\cline { 2 - 3 } & Serial Computing & Parallel Computing & \\
\hline A & 381.41 & 109.6 & 3.480018 \\
B & 401.72 & 443.28 & 0.906244 \\
C & 354.13 & 376.47 & 0.940659 \\
D & 421.65 & 113.46 & 3.716288 \\
E & 413.79 & 112.13 & 3.69027 \\
F & 388.58 & 109.78 & 3.539625 \\
G & 417.36 & 113.04 & 3.692144 \\
Total & 2778.64 & 1377.76 & 2.016781 \\
\hline
\end{tabular}

Figure 7 illustrates the wind energy curtailment rate and annual benefit brought by TED under different scenarios. The figure presents that, with the increase of TED capacity the wind energy curtailment rate drops remarkably, while, with different $P_{\text {sto }}-P_{\text {boiler }}$ proportions, the rate shows individual variation, which the higher the $P_{\text {boiler }}$ occupancy the faster the wind energy curtailment rate declines. That is the primary reason the electric boiler not only provides an outside heating source for the heating system, but absorbs electricity from the power system to promote the electric load level during hard peak shaving periods. Also, the wind energy curtailment rate shows more sensitivity to the change of heat storage capacity when $P_{\text {sto }}$ accounts for the majority of TED capacity. That is because the heating storage capacity limit plays an important role in influencing the effect of heating storage. 

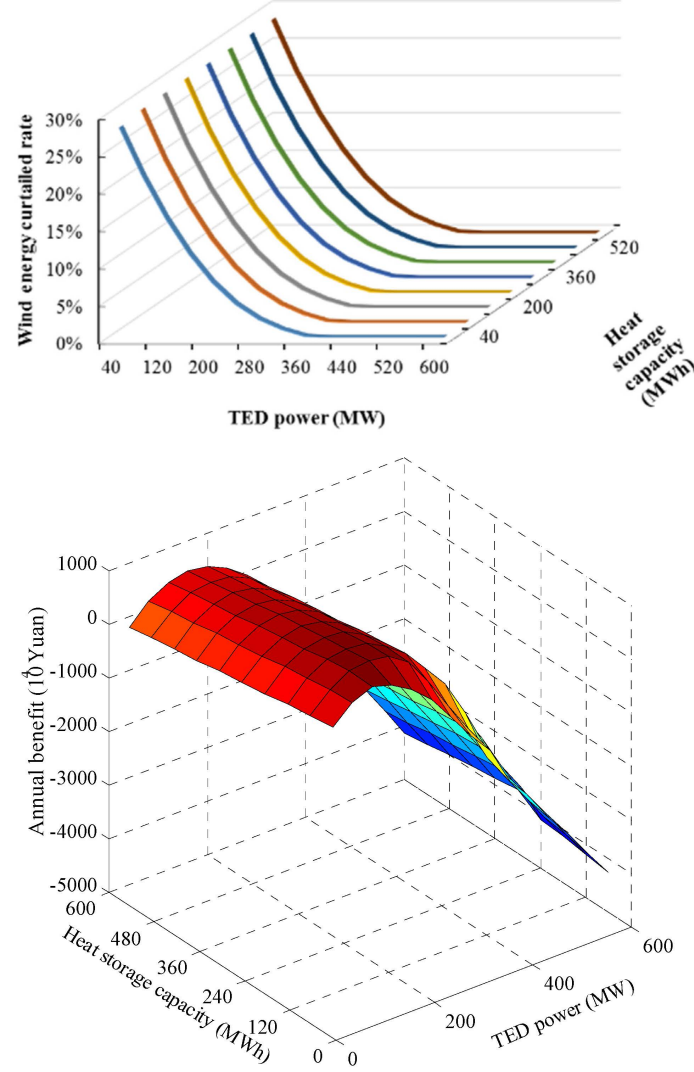

(a)
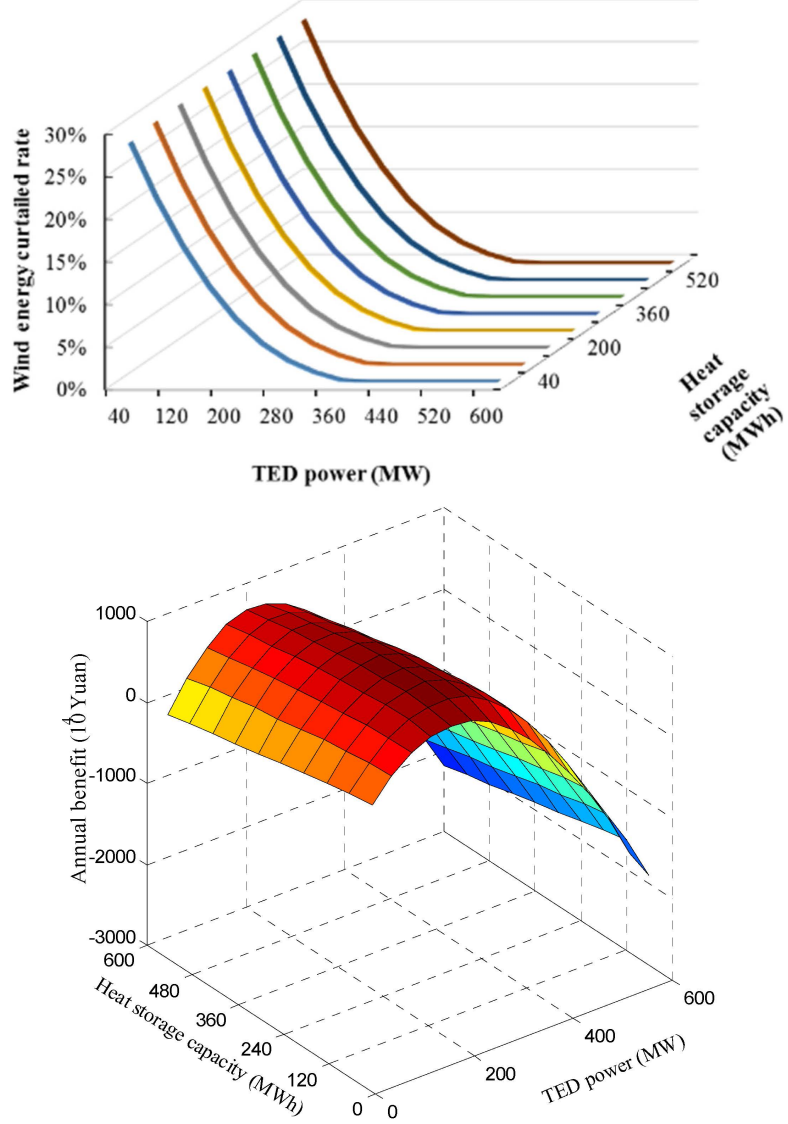

(b)

Figure 7. Cont. 

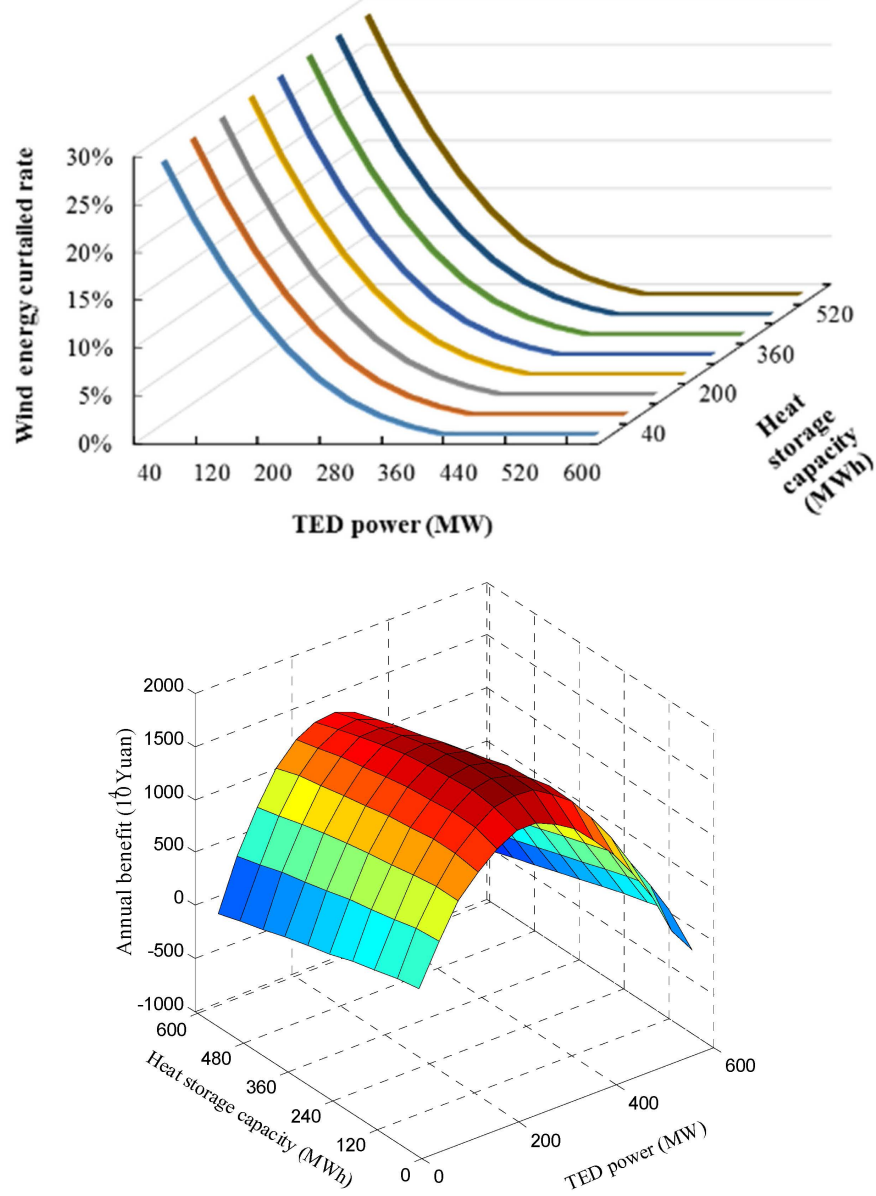

(c)
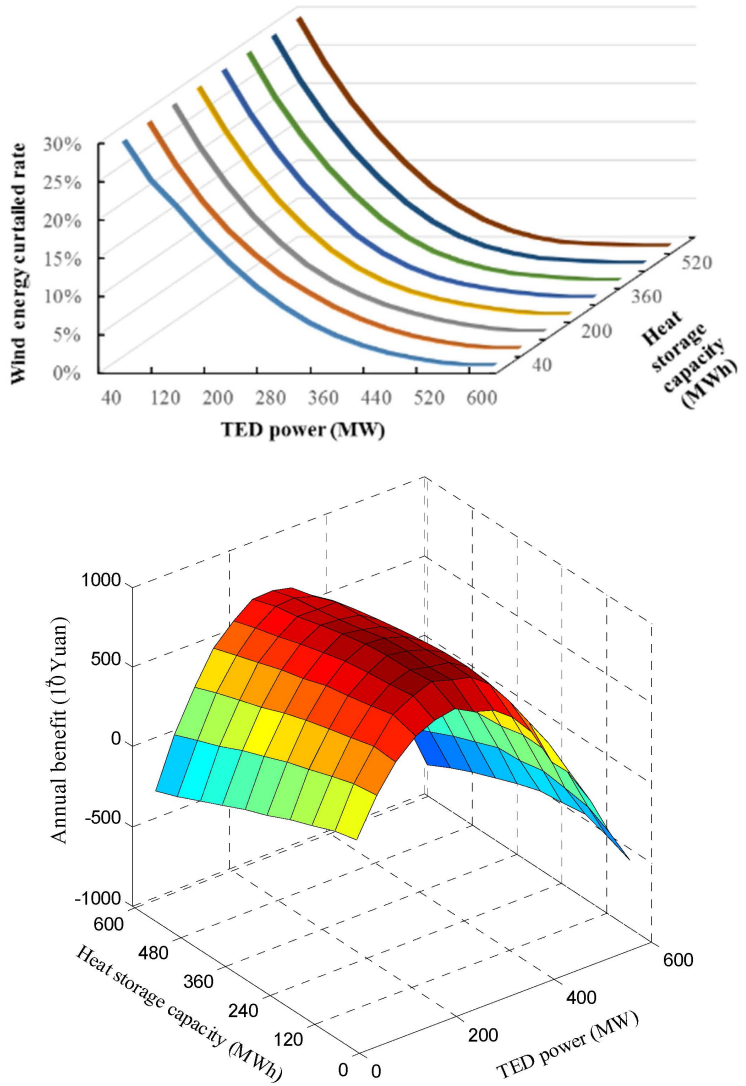

(d)

Figure 7. Cont. 

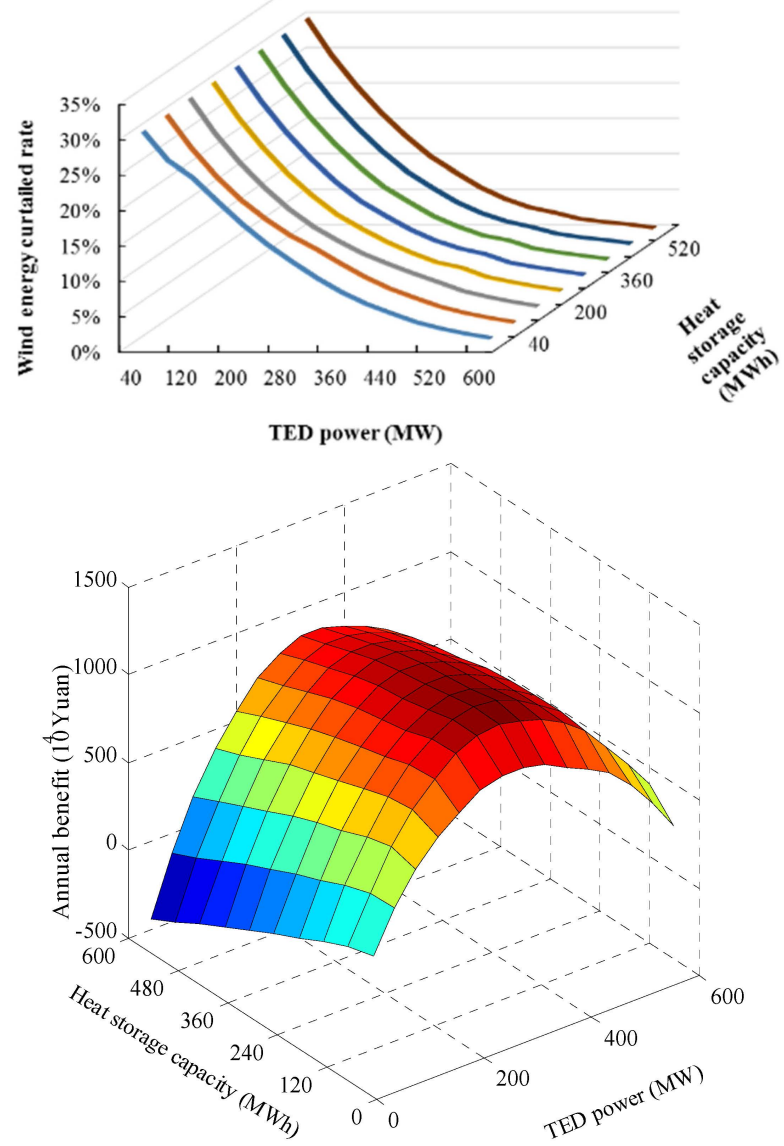

(e)
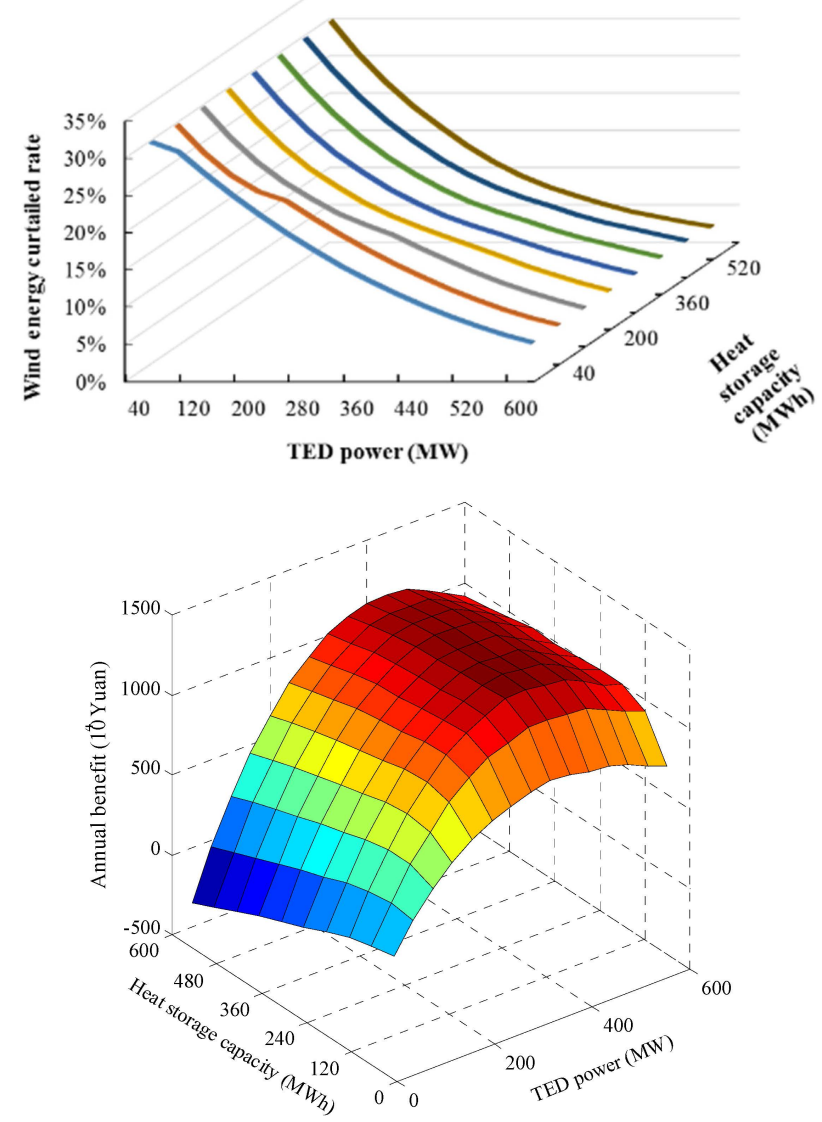

(f)

Figure 7. Cont. 

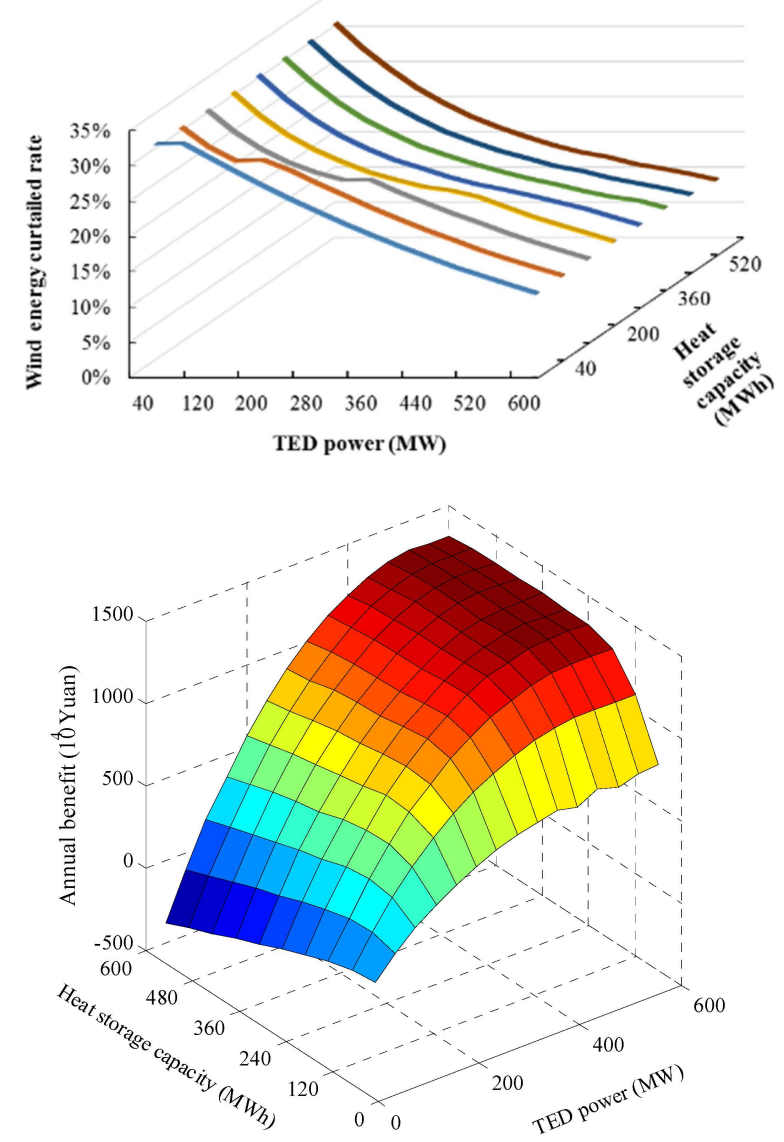

(g)

Figure 7. The wind energy curtailment rate and annual benefit bring by TED under different scenarios. (a) $P_{\text {sto }}: P_{\text {boiler }}=5: 35$; (b) $P_{\text {sto }}: P_{\text {boiler }}=10: 30$; (c) $P_{\text {sto }}: P_{\text {boiler }}=15: 25 ;$ (d) $P_{\text {sto }}: P_{\text {boiler }}=20: 20$; (e) $P_{\text {sto }}: P_{\text {boiler }}=25: 15 ;$ (f) $P_{\text {sto }}: P_{\text {boiler }}=30: 10 ;\left(\right.$ g) $P_{\text {sto }}: P_{\text {boiler }}=35: 5$.

Figure 8 illustrates the construction of annual incomes and costs of TED with the maximum profit under different electric boiler and storage output power ratios.

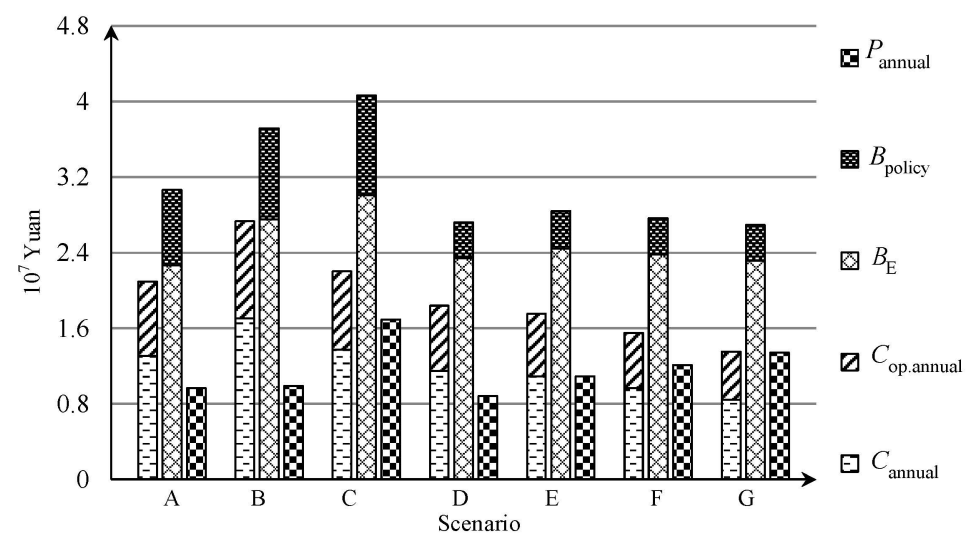

Figure 8. The annual costs, incomes and profits of TED.

Figure 9 illustrates the total initial investment of TED with maximum annual benefit under different scenarios. Because of the high price of electric boiler per unit power capacity, the electric boiler investment accounts for a considerable proportion of the total initial investment, and for similar reasons, the cost of power transformer equipment represents a significant amount of the total initial 
investment, while, both of them decrease with the raise of the $P_{\text {sto }}$ to $P_{\text {boiler }}$ proportion. Although, the cost on heat storage plays an minor role in the total initial investment due to low the per unit pricy, the heat storage needs a relatively large floor space, thus, with the raise of the $P_{\text {sto }}$ to $P_{\text {boiler }}$ proportion, the construction cost of the workshop $C_{\mathrm{ws}}$ occupies a vaster part of the total initial investment.

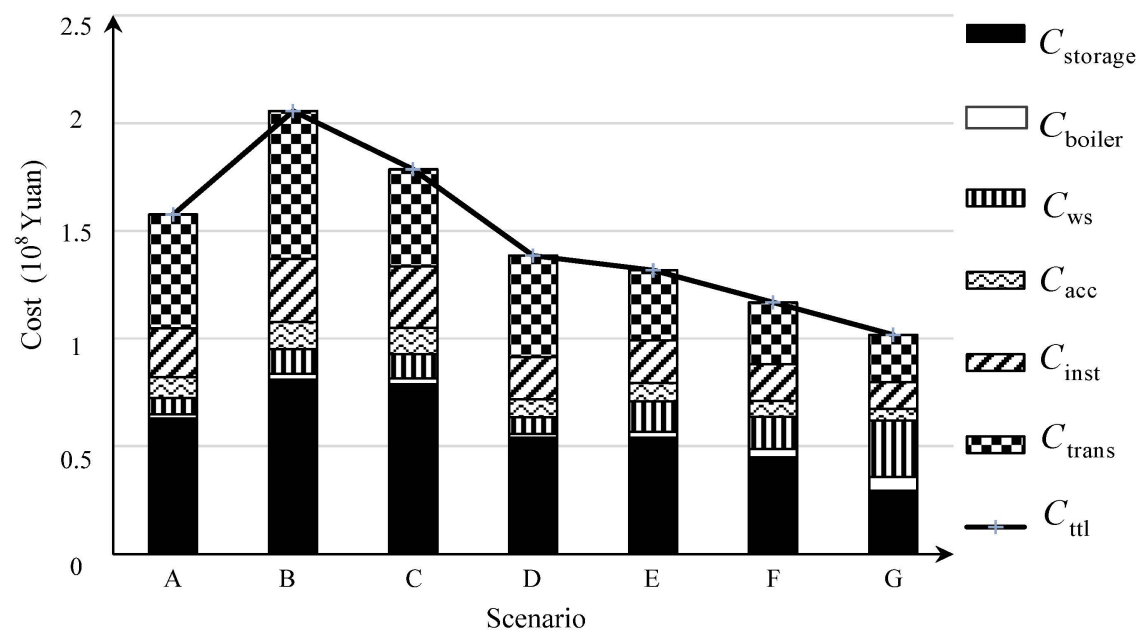

Figure 9. The initial investment of TED under different scenarios.

Table 4 demonstrates the maximum annual profit and TED allocation under different scenarios, from which it could be determined that, taking the maximum profit as the optimal object, under Scenario B the wind power curtailment could be avoid the most. Scenario B could attain the maximum annual profit, and the wind power curtailment could be fairly controlled, while the initial investment is the highest among all scenarios. The initial investment in Scenario $G$ is the lowest and could achieve a pretty good profit, but the effectiveness of wind power curtailment avoidance is not satisfactory.

Table 4. The maximum annual profit and TED allocation under different scenarios.

\begin{tabular}{cccccc}
\hline Scenario & $\begin{array}{c}\text { TED Power } \\
\text { (MW) }\end{array}$ & $\begin{array}{c}\text { Heat Storage } \\
\text { Capacity } \\
\text { (MWh) }\end{array}$ & $\begin{array}{c}\text { Maximum } \\
\text { Annual Profit } \\
\left(\mathbf{1 0}^{\mathbf{4}} \text { Yuan) }\right.\end{array}$ & $\begin{array}{c}\text { Wind Power } \\
\text { Curtailment Rate }\end{array}$ & $\begin{array}{c}\text { Wind Power } \\
\text { Consumption by TED } \\
\text { (GWh) }\end{array}$ \\
\hline A & 160 & 115 & 967.5298 & $11.2 \%$ & 38.87617 \\
B & 245 & 180 & 985.908 & $5.7 \%$ & 48.48795 \\
C & 280 & 185 & 1690.164 & $6.8 \%$ & 46.51416 \\
D & 240 & 120 & 881.7941 & $12.2 \%$ & 37.14052 \\
E & 320 & 175 & 1089.603 & $13.9 \%$ & 34.08095 \\
F & 400 & 240 & 1211.144 & $17.0 \%$ & 28.71823 \\
G & 525 & 415 & 1339.6209 & $22.1 \%$ & 19.79877 \\
\hline
\end{tabular}

\section{Conclusions}

In order to relieve the heavy wind power curtailment during the heating season in northern China areas, this work proposes a thermal-electric decoupling technology to enhance the peak shaving capacity of CHPs and increase the power load level during the wind power surplus period. A national economic mathematical model of thermal-electric decoupling is developed which takes CHPs, non-CHP units, wind farms and the heating network into account. An improved parallel conjugate gradient method is adopted to optimize the allocation of TED. Numerical simulation is given to verify the effectiveness of the algorithm and prove that the installation of TED can both bring down the wind power curtailment rate and produce a considerable economic profit and environmental revenue. With an allocation of TED by $280 \mathrm{MW}$ rated power and $185 \mathrm{MWh}$ heat storage capacity, 
the annual economic profit could be the maximum, which about 16.9 billion Yuan, and the wind power curtailment rate could be decreased to $6.8 \%$ from approximately $35 \%$.

Acknowledgments: This work has been supported by National Key Technology R\&D Program (2015BAA01B01), National High Technology Research and Development Program of China (863 Program) (2011AA05A105), and the National Natural Science Funds (50877014).

Author Contributions: Shuang Rong, Zhimin Li and Weixing Li checked and discussed the simulation results. Shuang Rong confirmed the series of simulation parameters and arranged and organized the entire simulation process. Zhimin Li participated in establishing the simulation model. Weixing Li revised the paper. Yong Sun and Taiyi Zheng made many useful comments and simulation suggestions. In addition, all authors reviewed the manuscript.

Conflicts of Interest: The authors declare no conflict of interest.

\section{Nomenclature}

\begin{tabular}{|c|c|}
\hline $\mathrm{CHP}$ & combine heat and power \\
\hline TED & thermal-electric decoupling \\
\hline$B_{\text {annual }}$ & annual incomes of TED \\
\hline$B_{\mathrm{E}}$ & annual fuel savings by lower electric power output \\
\hline$B_{H}$ & annual fuel saving by less heat supply \\
\hline$B_{\text {policy }}$ & annual policy incomes \\
\hline$C_{\mathrm{acc}}$ & cost of attached facilities \\
\hline$C_{\text {annual }}$ & annual cost of TED \\
\hline$C_{\text {boiler }}$ & investment on electric boilers \\
\hline$C_{\mathrm{E}}$ & annual electric energy cost on system electric capacity tariff \\
\hline$C_{\mathrm{HR}}$ & annual productive labor cost \\
\hline$C_{\text {inst }}$ & system installation and commissioning cost \\
\hline$C_{\text {MM\&SS }}$ & annual system maintaining and service cost \\
\hline$C_{m, n}$ & heating to electric power output ratio of $\mathrm{CHP}$ unit $n$ under backpressure condition \\
\hline Cop.annual & annual operation cost \\
\hline$C_{\text {storage }}$ & cost of heat storage \\
\hline$C_{\text {trans }}$ & investment on electric transformation equipment \\
\hline$C_{\mathrm{ttl}}$ & initial investment of TED \\
\hline$C_{\text {ttl.annual }}$ & annual investment cost discount to present equivalent annual worth \\
\hline $\mathrm{C}_{\mathrm{ws}}$ & construction cost of workshops \\
\hline$D_{\text {bank }}$ & bank discount rate \\
\hline$E_{\text {saved }}$ & consumed wind energy by TED during the heating season \\
\hline Esto & storage capacity of heat storage within TED \\
\hline$H_{z, t}^{\mathrm{HB}}$ & heating power output of heating boiler unit $z$ at moment $t$ \\
\hline$H_{n, t}^{\mathrm{CHP}}$ & heating power output of CHP unit $n$ \\
\hline$H_{k, t}^{\text {TED,sup }}$ & heating power output of TED unit $k$ at moment $t$ \\
\hline$H_{k, t}^{\mathrm{TED} \text {,sto }}$ & input heating power of TED unit $k$ at moment $t$ \\
\hline$\overline{H_{n}}$ & lower bounds of heating power output of heat source $n$ \\
\hline$\underline{H_{n}}$ & lower bounds of heating power output of heat source $n$ \\
\hline$\overline{H_{\mathrm{u}, n}}$ & upper bond of heating power ramp rate of $\mathrm{CHP}$ unit $n$ \\
\hline$\underline{H_{\mathrm{d}, n}}$ & lower bonds of heating power ramp rate of $\mathrm{CHP}$ unit $n$ \\
\hline$H_{\text {season }}$ & maximum heat load during heating season \\
\hline$K_{n}$ & a constant \\
\hline$L_{\mathrm{E}, t}$ & system electric load and power transfer to outside at cross-section \\
\hline
\end{tabular}




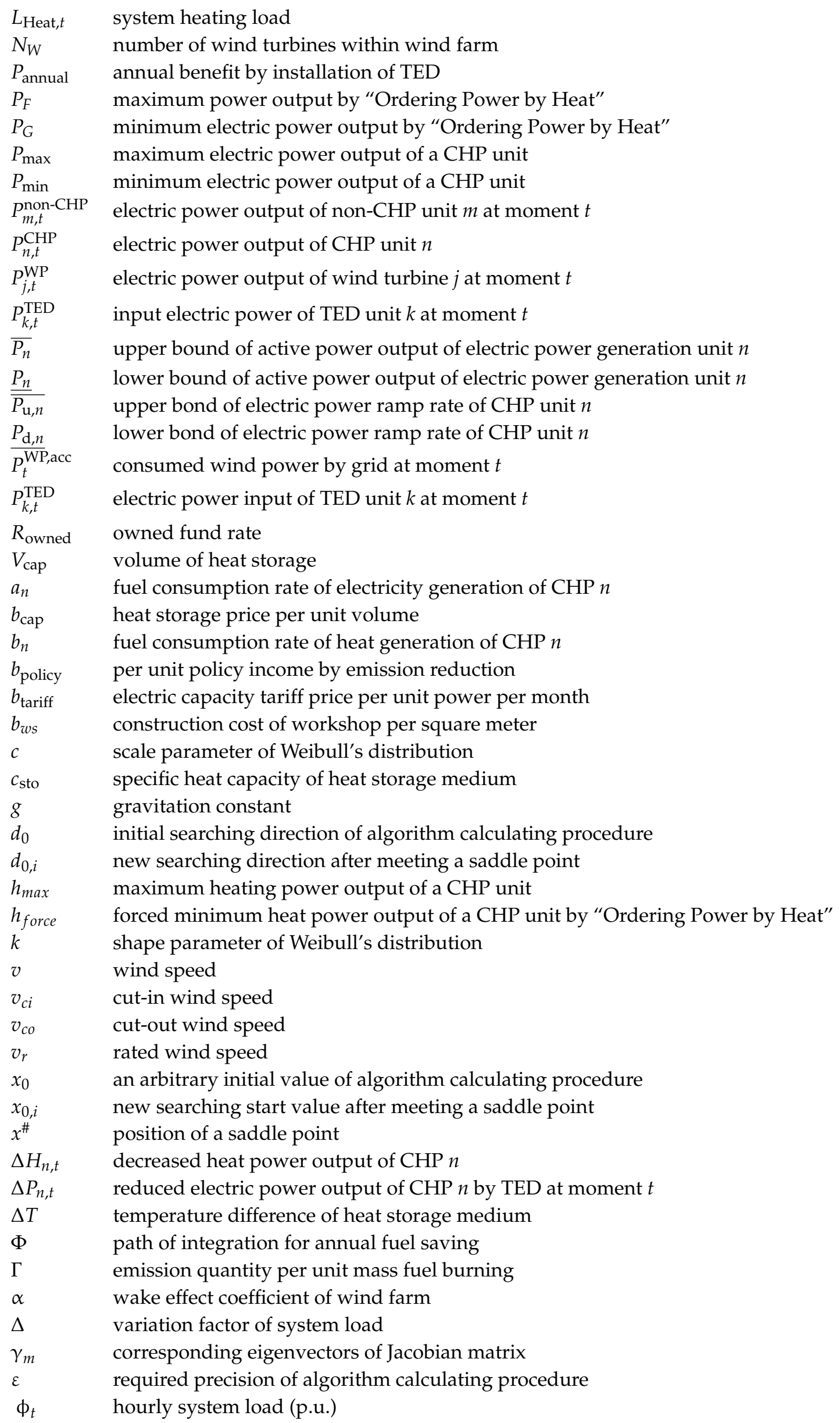


$\xi_{\mathrm{H}, n} \quad$ daily heat load (p.u.) during heating season

$\lambda_{m} \quad$ eigenvalues of Jacobian matrix

$\rho_{\text {sto }} \quad$ density of heat storage medium

$\xi_{\mathrm{E}, n} \quad$ weekly system electric load (p.u.) during heating season

\section{References}

1. Yang, X.; Song, Y.H.; Wang, G.H. Comprehensive Review on the Development of Sustainable Energy Strategy and Implementation in China. IEEE Trans. Sustain. Energy 2010, 22, 57-65. [CrossRef]

2. Liang, R.H.; Liao, J.A. Fuzzy-Optimization Approach for Generation Scheduling with Wind and Solar Energy Systems. IEEE Trans. Power Syst. 2007, 4, 1665-1674. [CrossRef]

3. Zhang, X.H.; Zhao, J.Q.; Chen, X.Y. Multi-objective Unit Commitment Fuzzy Modeling and Optimization for Energy-saving and Emission Reduction. Proc. CSEE 2010, 22, 71-76.

4. The Renewable Energy Law of China. Available online: http://www.gov.cn/fwxx/bw/gjdljgwyh/ content_2263069.htm (accessed on 15 October 2015).

5. Global Wind Energy Council. Global Wind Report. Available online: http://www.gwec.net/publications/ global-wind-report-2/global-wind-report-2014-annual-market-update/ (accessed on 15 October 2015).

6. Wang, Z.Y.; Shi, J.L.; Zhao, Y.Q. The Roadmap of China Wind Power Development 2050, 1st ed.; Energy Research Institute, National Development and Reform Commission: Beijing, China, 2011; pp. 32-36.

7. Wang, X.H.; Qiao, Y.; Lu, Z.X.; Ding, L.; Shao, G.H.; Xu, X.W.; Hou, K.Y. A Novel Method to Assess Wind Energy Usage in the Heat-supplied Season. Proc. CSEE 2015, 9, 2112-2119.

8. Ibrahim, H.; Ilinca, A.; Perron, J. Energy storage systems-characteristics and comparisons. Renew. Sustain. Energy Rev. 2008, 5, 1221-1250. [CrossRef]

9. Ibrahim, H.; Ilinca, A.; Perron, J. Comparison and analysis of different energy storage techniques based on their performance index. In Proceedings of the Electrical Power Conference 2007, Montreal, QC, Canada, 25-26 October 2007; pp. 393-398.

10. Nourai, A. Large-scale electricity storage technologies for energy management. In Proceedings of the Power Engineering Society Summer Meeting 2002, Chicago, IL, USA, 21-25 June 2002; pp. 310-315.

11. Kondoh, J.; Ishii, I.; Yamaguchi, H.; Murata, A.; Otani, K.; Sakuta, K.; Higuchi, N.; Sekine, S. Electrical energy storage systems for energy networks. Energy Convers. Manag. 2000, 7, 1863-1874. [CrossRef]

12. Elsobki, M.S. Implementation of an integrated resource planning strategy: An optimal based formulation for co-generation applications. In Proceedings of the 14th International Conference and Exhibition on Electricity Distribution: Part 1 Contribution, London, UK, 2-5 June 1997; pp. 5-10.

13. Fu, L.; Jiang, Y. Optimal Operation of Backpressure Units for Space Heating. Proc. CSEE 2000, 3, 81-84.

14. Huang, S.H.; Chen, B.K.; Chu, W.C. Optimal operation strategy for cogeneration power plants. In Proceedings of IEEE Industry Applications Conference, Seattle, WA, USA, 3-7 October 2004; pp. 2057-2062.

15. Guo, T.; Henwood, M.I.; van Ooijen, M. An algorithm for combined heat and power economic dispatch. IEEE Trans. Power Syst. 1996, 4, 1778-1784. [CrossRef]

16. Long, H.Y.; Ma, J.W.; Wu, K. Energy conservation dispatch of power grid with mass cogeneration and wind turbine. Electr. Power Autom. Equip. 2011, 11, 18-22.

17. Jiang, X.L.; Lu, Q.Y.; Xie, G.N. A Preconditioned Parallel Method for Solving Saddle Point Problems. Appl. Math. Mech. Engl. 2014, 9, 1011-1019.

18. Rong, S.; Li, Z.M.; Li, W.X. Investigation of the Promotion of Wind Power Consumption Using the Thermal-Electric Decoupling Techniques. Energies 2015, 8, 8613-8629. [CrossRef]

19. Yang, M.; Li, Z.M.; Cao, D.Y. Parallel algorithm for solving large-scale dense linear system on CUDA. Comp. Engl. Appl. 2011, 32, 27-30.

20. Yang, M.; Sun, C.; Li, Z.M.; Cao, D.Y. An improved sparse matrix-vector multiplication kernel for solving modified equation in large scale power flow calculation on CUDA. In Proceedings of the Power Electronics and Motion Control Conference (IPEMC), Harbin, China, 2-5 June 2012; pp. 2028-2031. 